\title{
ANÁLISIS DE REDES SOCIALES POR GEO-MAPA: LAS RELACIONES INSTITUCIONALES DE LA UFMT/BRASIL
}

\author{
Adilson Luiz Pinto* \\ Departamento de Ciencia de la Información. Universidade Federal de Santa Catarina.
}

Resumen: Se pretende identificar la representación científica de la Universidade Federal do Mato Grosso (UFMT) en relación a su participación en los productos Google Earth y Google Maps, en los que se han entrelazado aquellas relaciones más importantes con más de 5 trabajos publicados en conjunto dentro de la Web of Science. Para la recuperación de datos se ha utilizado el método de filiación institucional (C1); mientras que el tratamiento se ha hecho en Excel a través de GPS Visualizer's para mapear las longitudes y latitudes. Posteriormente se generó una página para hospedar la estructura de las relaciones en .kml, lenguaje que es legible por Google Maps y Google Earth. En los análisis de redes sociales, la generación de mapas de relación está pasando por un cambio importante, pues hoy se ha convertido ya en tendencia identificar las relaciones en ámbito macro, siendo una de las forma de alcanzar la visibilidad mundial de una institución de investigación, respecto a sus colaboradores, averiguar los principales compañeros científicos y la transposición a escala de mapamundi, en el que la Universidade Federal de Mato Grosso consiguió firmar buenos acuerdos científicos en Brasil (con participación de las Universidades: de Sao Paulo, Federal do Rio de Janeiro, Federal de Viçosa, Unicamp y Unesp) y fuera, con el Max Planck Institute of Limnology y la Darmstadt University of Technology, que han resultado los más destacados en la escala de productividad. Estas relaciones han sido constantes en Medicina; Agricultura; Ingenierías; Física; Química y Zootecnia.

Palabras clave: geo-mapas; UFMT; análisis de redes sociales; Google Maps; Google Earth.

Title: SOCIAL NETWORK ANALYSIS BY GEO-MAP: THE RELATIONS OF THE UFMT / BRAZIL.

Abstract: This study has the objective to identify the scientific representation of the Federal University of the Mato Grosso (UFMT), relationed to the participation on the programs Google Earth and Google Maps, where the most important relationships are showing with more than 5 articles published on Web of Science. For recover data, was used a search by the institutional affiliation (C1) while the treatment was done on Excel using GPS Visualizer's, for to map the longitudes and latitudes. At a later time was created a page for to support the structure on the format .Kml, language that can be read by the Google Maps and Google Earth. On the network analysis, the origination of relation map it passes for big changes becoming a trend for to indentify relations in a higher level, one of the ways to gain global visibility of a research institution related to the employees, scientific colleague, finding the key on the scale of transposition that the Federal University of Mato Grosso could sign importants agreements in their scientific cooperation (with the participation of the Universities: USP, UFRJ, Federal de Visçosa, Unicamp and Unesp) and on the foreign countries with Institute Max Planck of limnology and from Darmstadt Technology University, whom had been the most prominent on the productive scale. In scientific areas was detached in relations on Medicine, Agriculture, Engineering, Physics, Chemistry and Animal Production.

Keywords: geo-maps; UFMT; analysis social network; Google Maps; Google Earth.

*adilson@cin.ufsc.br

Recibido: 18/10/2012; 2a versión: 14/01/2013; aceptado: 28/01/2013.

PINTO, A.L. Análisis de redes sociales por geo-mapa: las relaciones institucionales de la UFMT/Brasil. Anales de Documentación, 2013, vol. 16, $\mathrm{n}^{\circ} 1$.

Disponible en: http://dx.doi.org/10.6018/analesdoc.16.1.161051 


\section{INTRODUCCIÓN.}

La forma por la que se ha pasado a medir y representar la visibilidad científica ha alcanzado unos escenarios radicalmente diferentes a los acostumbrados hasta hace poco tiempo en que iba unida exclusivamente a indicadores en forma de tablas y gráficos. El cambio más notorio ha venido de la mano de un nuevo modo de generar estos índices. No es otro que la cientografía, que es la representación de la visibilidad mediante mapas de relaciones (Turuget, 1989).

En el contexto científico-tecnológico, la elaboración de mapas de relaciones mediante el análisis de redes sociales ha fijado el camino a la hora de reflejar el perfil de las instituciones en lo relativo a la colaboración y sus agentes, e incluso a la hora de plasmar las coocurrencias que pueden darse en la producción y en las citas.

Estos índices permiten presentar descriptivamente, de manera simultánea, las diferentes variables o las múltiples relaciones que pueden observarse en los documentos, aspecto este muy cercano a lo propuesto para la comprensión de la bibliometría (Sanz Casado; Martín Moreno, 1998), estudio de la cuantificación de documentos y bibliografias.

La medición se ha convertido en punto cardinal para la ciencia y la tecnología (CyT) de un país (Holbrook, 1992), pues suministra informaciones prominentes, no convencionales, de forma esencial e inteligible para este universo. Así pues, los indicadores de CyT son medidas cuantitativas que buscan representar conceptos muchas veces intangibles dentro de este entorno, tales como el volumen de inversiones en CyT en relación al PIB, la proporción de profesionales de nivel superior en el mercado de trabajo, la cantidad de másteres y doctores en relación a todos los titulados, los coeficientes técnicos de los investigadores por cada 100 mil habitantes, y los artículos publicados en revistas científicos (Velho, 1990 y 1998) o el modo en que se desarrollan los colegios invisibles.

Sobre este último punto, reforzado por el análisis de red social, se reafirma nuestra propuesta. De manera que buscamos determinar lo que un análisis de red social (ARS) y sus esferas pueden contribuir para medir la actividad de CyT.

Desde nuestra perspectiva, ARS refleja las interacciones que se producen entre los integrantes que están conectados horizontalmente, bien directamente o a través de los que se sitúan en proximidad. El conjunto resultante de esta relación es una malla de múltiples vectores, que muestra conexiones indeterminadas en todos los sentidos espaciales, sin que ninguno de sus nodos pueda ser considerado principal o central, ni representante de los demás, por lo que no cuenta con un nodo cabecera. Lo que muestra es un esfuerzo colectivo a la hora de plasmar y alcanzar determinados objetivos (Withaker, 1998).

Su empleo en CyT pasó desde la mera representación de la colaboración hasta una configuración más sofisticada, como la propuesta por Leydesdorff y Persson (2010), a la hora de formatear datos de la Web of Science y trabajar con la ubicación de los autores, 
estableciendo un mapa de relaciones dentro de la gran plataforma de representación del mapamundi, siempre mediante el uso de herramientas Google. Nuestra propuesta persigue una representación similar, pero limitada a instituciones de investigación, también con diferencias en la forma de generar las matrices y los propios mapas, cuyo diseño refleja los resultados obtenidos.

Desde estos presupuestos, nos propusimos trabajar con los trazos digitales y sus conexiones dentro de una realidad indicativa, tratando de forma sencilla la representación de las redes sociales (Farman, 2010) a partir del empleo de Google Maps y Google Earth. Lo específico era manejar informaciones en lo tocante a la dirección laboral de los investigadores dentro de la Web of Science. Proceso este distinto del propuesto por Bigham (2009) que estudió la colaboración sobre datos mediante aplicativos de Google para representar la gran complejidad de los accidentes de tráfico en California. Sin embargo, el proceso de generación de los mapas se ha enfrentado de modo bastante similar, pues se ha basado en determinar las longitudes y las latitudes. Otra diferencia consistió en que la aplicabilidad de Bigham se filtró mediante ArcGIS $^{1}$ y tenía como finalidad la vigilancia de personas, mientras que la nuestra se hizo en GPS Visualizer's ${ }^{2}$ y estuvo enfocada a evidenciar las relaciones existentes entre investigadores pertenecientes a la Universidade Federal de Mato Grosso.

Como objetivo general, se ha querido mostrar una nueva aplicación de trabajo con geo-mapas y sus representaciones para el medio académico, enfocada a exhibir las colaboraciones existentes y como se disponen dentro del mapamundi generado por Google Maps y Google Earth.

Mientras que se situarían como objetivos específicos:

- Averiguar cómo se distribuye la colaboración científicas en una dimensión geográfica universal;

- Identificar quienes son los principales colaboradores de la Universidade Federal de Mato Grosso (Brasil); e

- Identificar, dentro de la geografía de los mapas, cuestiones relacionadas con clusters de colaboración, tanto aglomeraciones como perfiles que se dan en las investigaciones del estado de Mato Grosso y qué compañeros científicos se muestran.

\section{METODOLOGÍA.}

Para representar las relaciones en visibilidad geográfica, a la vez que los aspectos relativos a la medición de los datos sobre colaboración, se ha optado por emplear una metodología de carácter descriptivo tanto cualitativa como cuantitativa.

El proceso inicial consistió en delimitar el universo de la investigación y los campos que serían atendidos por la muestra, de manera que optamos, entre los campos que contiene la Web of Science, por considerar solamente el campo de autoría, representado 
dentro de la base datos como $\mathrm{C} 1$, en el que se informa sobre la dirección laboral de cada uno de los autores de un estudio.

El siguiente paso fue determinar las relaciones existentes dentro del universo de artículos que representaban la producción de la UFMT de 1975 hasta 2010, con una subfase para registrar aquellas que ofrecían una relación superior a 5 colaboraciones con la UFMT. Con todas las relaciones posibles, por encima de 5 participaciones, se confeccionó un fichero en Excel, en cuatro columnas, en el que se identificó la frecuencia de co-ocurrencia, el nombre de las instituciones, sus ciudades, provincias y país. Con estas informaciones se migró a otro fichero en una sola columna, con el objetivo de marcar una ruta geográfica, a partir del nombre institucional.

Después se detallaron todos los datos en una única columna, mediante GPS Visualizer's, determinando como entrada los nombres institucionales con sus ciudades y países, y recuperando como salida los códigos de latitud y longitud de las instituciones que se han mostrado como compañeras científicas de la UFMT.

En la secuencia se identificaron algunos parámetros de los datos, con objeto de que el contenido pudiera ser leído por las herramientas de Google (Maps y Earth), de manera que se utilizó el estándar [T,-15.5989173,-56.094894,"UFMT, Cuiaba, MT, Brazil","Cuiabá MT, Brazil",,,100,0] para orientar las rutas, que quedaron determinadas por el tipo de relación, la latitud, la longitud, el nombre de la institución, el color de las relaciones y los formatos de identificación de las relaciones.

El siguiente paso fue convertir el fichero en Excel en una extensión que admitiesen las herramientas de Google. Se pasaron así los datos a .kml, para lo que se utilizó Earth Point. Este proceso sirvió para identificar los mapas en Google Earth, sin embargo no valió para generar los mapas en Google Maps, por lo que se decidió utilizar otra técnica y se decidió colgar este contenido en una página web pero con descripción para .kml, pues Google Maps sólo identifica direcciones y links. El 23/05/2011 se creó un link para la exportación de los datos: <http://70.36.96.13/EarthPointExcel20.kml> que después fue vislumbrado como: $\quad<$ http://maps.google.com.br/maps/ms?hl=ptBR\&ie $=$ UTF $8 \&$ msa $=0 \&$ msid $=215323711773300844089.0004 a 3 \mathrm{fc} 186459 \mathrm{a} 5 \mathrm{bef} 82 \& 1 \mathrm{l}=59$ $.534318,-161.71875 \& \mathrm{spn}=178.148744,70.3125 \& \mathrm{z}=0>$. En la secuencia se muestra un fragmento del código del documento en . kml.

Anales de Documentación, 2013, vol. 16, nº 1 


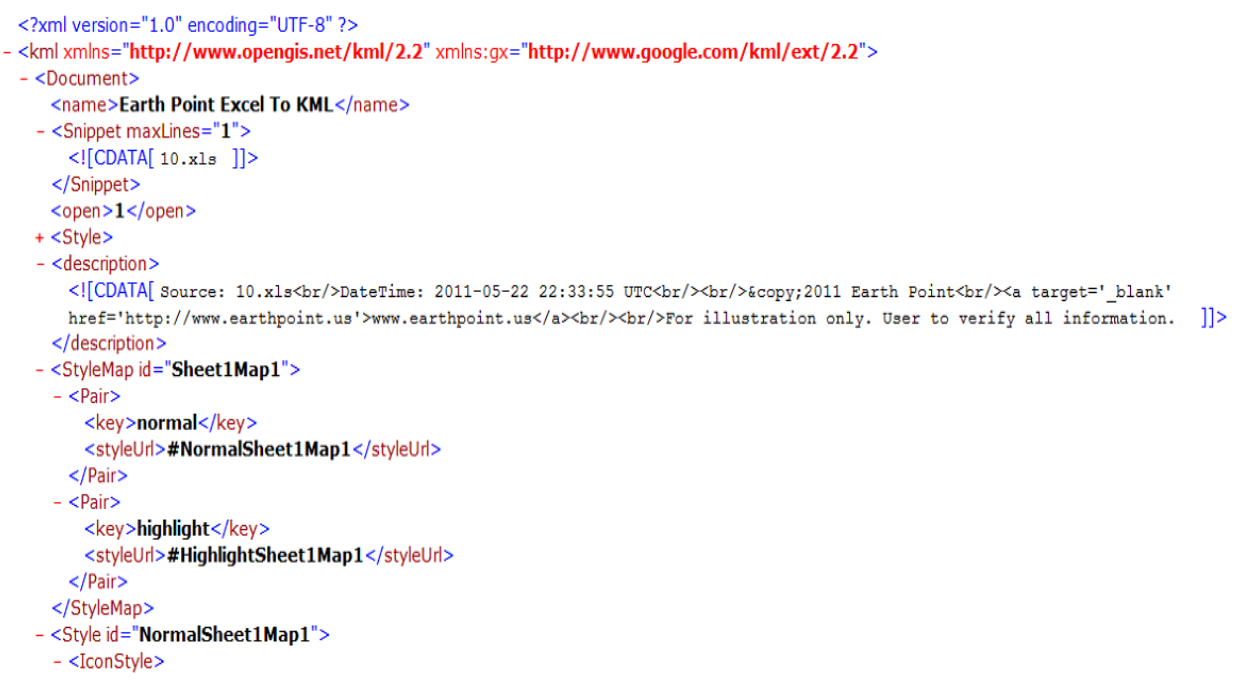

\section{ESTADO DEL ARTE.}

Se buscaba dar con una estrategia que permitiese generar mapas de relaciones en internet a través de Google Maps y Google Earth. Sin embargo este hecho no califica por sí mismo un estudio aplicado. De ahí que se decidiera generar esos índices para una universidad brasileña, pues es más práctico aplicar un estudio de caso que determinar sólo una metodología posible. La metodología usada para el análisis de redes sociales muestra carácter innovador, aunque se hayan estudiado mucho las redes debido a la gran posibilidad de validación científica que ofrecen.

La diversificación es tan amplia que conviene destacar algunas propuestas relevantes en relación al análisis de redes sociales y su visualización virtual, como la propuesta de Crosby (1997) que hace de la visualización uno de los dos factores responsables del desarrollo explosivo de todos los de la ciencia moderna. Freeman (2000), corrobora sobre este fenómeno, diciendo que $[\ldots]$

Advances in measurement have been both rapid and regular in social network research. And, from the beginning, visual images have played a key role in the field. Images of social networks have provided investigators with new insights about network structures and have helped them to communicate those insights to others (Freeman, 2000, p. 1).

Existen muchos estudios de suma importancia para las redes, pero vamos a limitarnos a algunos actuales como el de Yang, Liu y Meloche (2010) que plantea un análisis webométrico de las 100 mejores universidades de la República de China, para el que utilizaron los vínculos de relación entre sus sitios web a través de sus componentes 
principales tales como compañeros de enlace e intermediación, con escala multidimensional y redes sociales como método de análisis.

Por lo común, las instituciones tienen una organización centralizada, pero existen ejemplos y aplicaciones de centralidad también en el caso de las personas. Dentro de los análisis de redes aparecen las personas con liderazgo, con variedad de tipos y ejemplos de casos para identificar los resultados asociados con cada tipo de red. La evaluación de las redes de liderazgo es un desafío, pues trabaja un enfoque que utiliza las métricas y la visualización para representar la estructura de las relaciones entre las personas, organizaciones, objetivos, intereses y otras entidades dentro de un sistema (Hoppe y Reinelt, 2010). También puede deberse a participación por mérito dentro de la escala científica, como la propuesta hecha por White (2001), sobre los Cameo's ${ }^{3}$ de la ciencia.

Los estudios que analizan las redes sociales parecen no tener límites, como la propuesta de Chin Wu e Ying Wu (2011) para desarrollar el Wikimap y aplicarlo al estudio de los contenidos libres de la enciclopedia multilingüe en la web (Wikipedia). Su propuesta vislumbra los indicadores para etiquetar las relaciones existentes entre los artículos y los temas para ayudar a que los usuarios identifiquen y encuentren la información que buscan. Para evaluar la eficacia del método que propone Wikimap, se realizó un estudio sobre 28 participantes en el que se pidió ejecutasen tareas simuladas de búsqueda en dos interfaces: la interfaz estándar de Wikipedia y la interfaz propuesta en Wikimap.

En contrapartida, hay también una notoria innovación del software aplicable a la representación de las redes sociales, como es el caso de GraphDice, un sistema de red de visualización multivariante para explorar el espacio de atributos que muestran las arrestas y los actores. El programa se basa en el sistema ScatterDice cuyo paradigma principal es la navegación multidimensional, y la ampliación con nuevos mecanismos para apoyar la exploración de la red en tareas generales y más concretas (Bezerianos; Chevalier; Dragicevic; Elmqvist y Fekete, 2010).

\section{RESULTADOS.}

Con la misma intención de aprovechar las aplicaciones y el desarrollo de métodos y programas se sitúa la representación de redes por determinación geográfica que se aplicará a continuación al caso práctico de la distribución de la colaboración científica en la Universidad Federal de Mato Grosso (UFMT) a través de su reflejo en la Web of Science.

Anales de Documentación, 2013, vol. 16, nº 1 


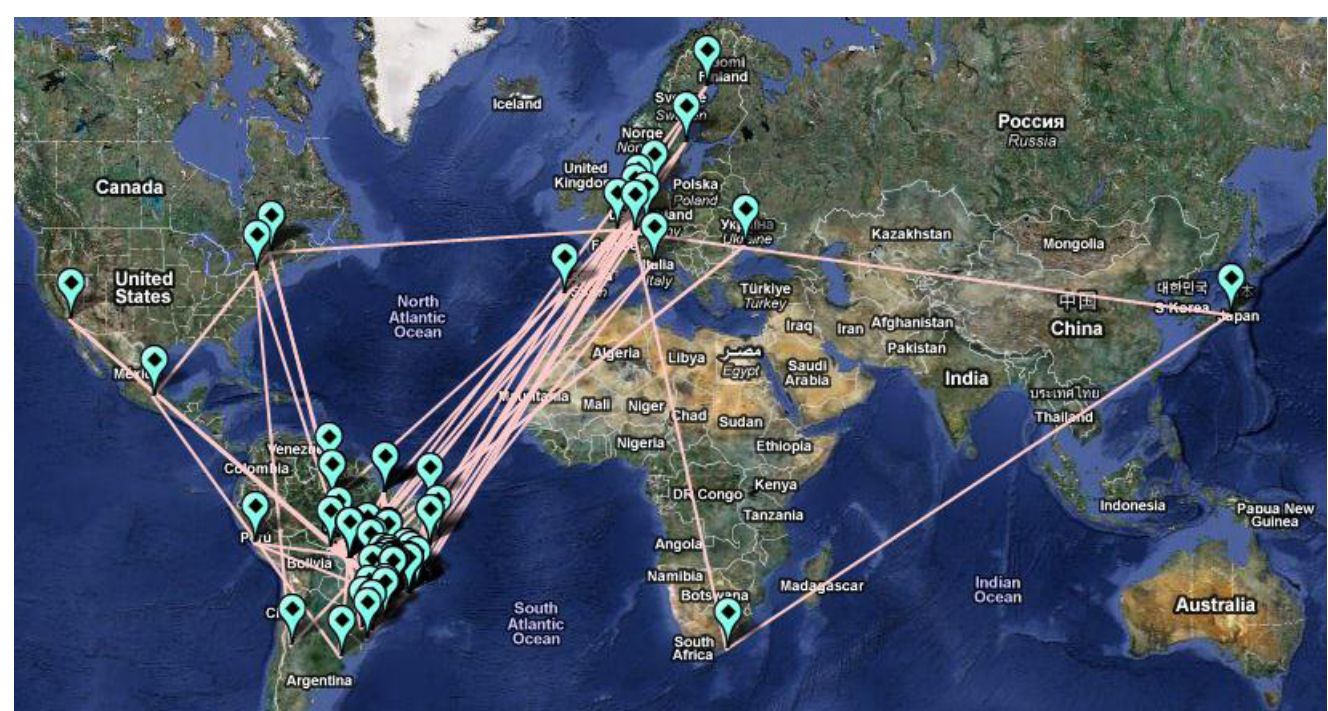

Figura 1. Distribución de las relaciones de la UFMT en el Google Maps a partir de datos de la Web of Science.

De partida, nuestra propuesta ha considerado la frecuencia absoluta de la UFMT, por tratarse de una institución educativa e investigadora que difunde sus logros principalmente en forma de artículos, y que ha repercutido positivamente las circunstancias favorables que la han situado como la institución más beneficiada en el estado de Mato Grosso (Brasil) en lo que respecta a la investigación científica. Ha retribuido así la inversión en investigación con que anualmente han dotado a esta institución las agencias de fomento.

Entre estos datos de productividad, especialmente en lo referente a la colaboración, se destacan algunos a la hora de considerar cómo las instituciones estatales han firmado sus hallazgos, de manera notoriamente enraizada entre la UFMT y las universidades brasileñas USP, UFRJ, UFV, UFLA, Unicamp y Unesp, pero también con las europeas Max Planck Institute Limnology y University Technical of Darmstadt.

Si consideramos las asociaciones científicas en general, a partir de las relaciones fijadas en las representaciones espaciales, puede afirmarse que la UFMT es el centro de las actividades en el Estado de Mato Grosso, a pesar de mostrar una influencia casi absoluta de la unidad de Cuiabá, lo que refleja también cuanto sucede en la vida económica y social en ese estado.

A la hora de cuantificar el principio de grado, que mide el grado de cada actor dentro de la red con independencia de la calidad de sus contactos, la UFMT llega a una frecuencia graduada en 251 relaciones directas, donde 96 de estas relaciones son directas con la USP. En la secuencia se detectaron 56 relaciones directas con la UNEMAT, seguida por la Unesp con 51, UFLA y UFG ambas con 35 relaciones cada una, UFRGS con 34, UNB con 32, finalmente UFV y Unicamp cada una con 29 grados de relación. 


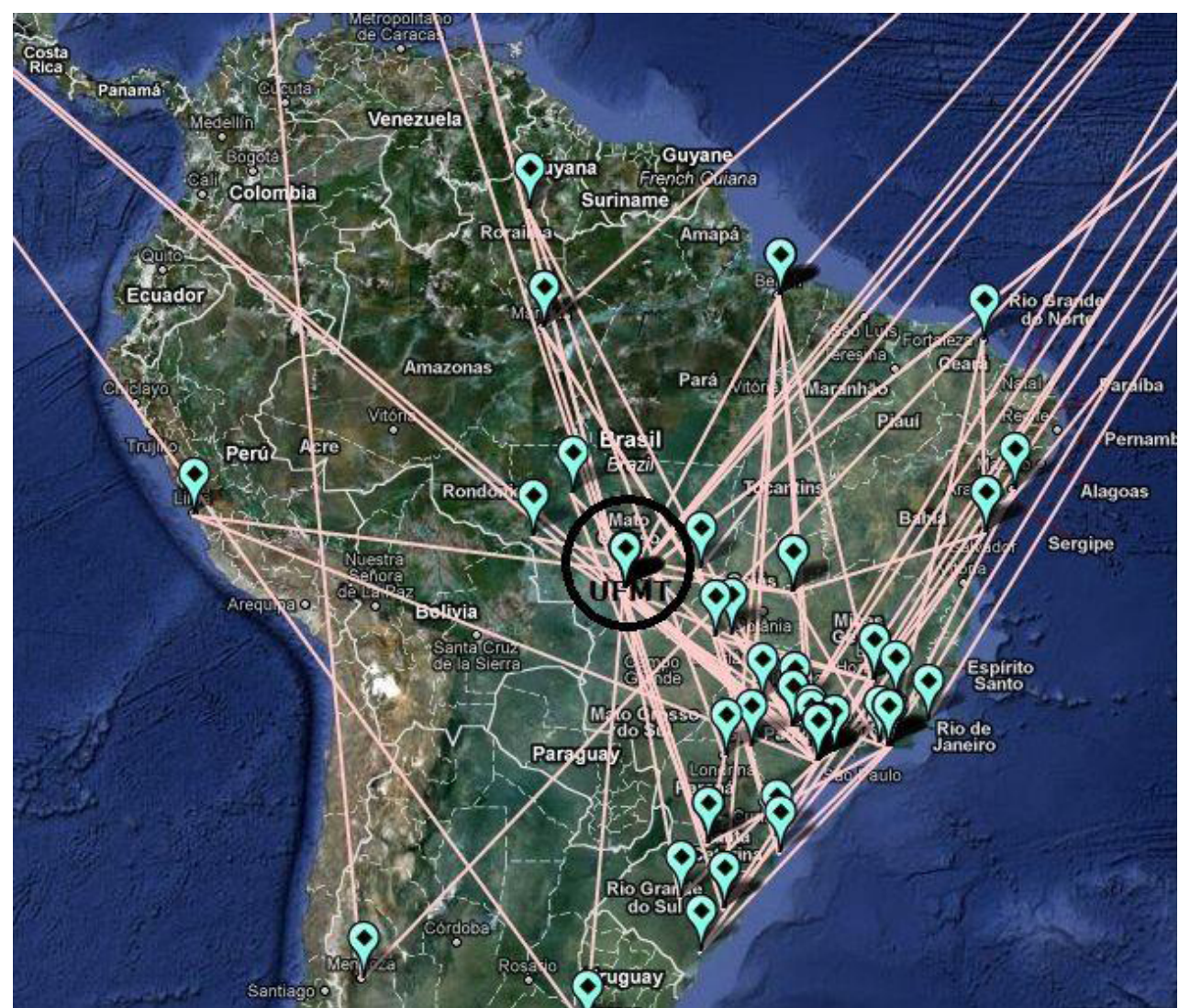

Figura 2. Distribución de las relaciones de la UFMT con las instituciones locales en el Google Maps en la Web of Science. Cada punto representa una institución.

Otra medida considerada en las visualizaciones por mapamundi ha sido la centralidad de intermediación, que muestra aquellos actores que tienen una posición de ventaja (o poder) dentro de la red, y que se representan siguiendo el camino más corto entre dos conjunto de actores, también conocido como tasa de intercambio. En este estudio aparece con alto relieve la intensa presencia sociométrica de la UFMT, con un total de 53.920 puntos de intermediación en la red, seguido por sus colaboradores más próximos como la USP, con 9892 puntos (directos e indirectos), y en orden decreciente UNEMAT con 8544, UFLA con 7305, UNESP con 6488, Centro de Información de UNB con 6229, NFB con 2287, UFRRJ con 1894, UFG con 1786, UFRGS con 1450, Embrapa Algodão con 1398, UNICAMP con 1013 y, por fin, CEFET con 969.

El análisis métrico también se aplicó a la cercanía central o proximidad a los enlaces en la red, que se representa por el grado en que un actor aparece cerca del resto de actores en la red, ya sea directa o indirectamente. La inversión de la suma de las distancias más 
cortas entre cada individuo y su proceso también se conoce como aplicación de las distancias geodésicas. En el caso de esta revisión los más destacados en la colaboración con la UFMT fueron: Hospital Garcia Horta, Hospital S. Francisco Xavier, Universidad Nova de Lisboa e Molecular Cancer Research Center (todos ellos con 159601 puntos de proximidad), seguidos por FAPEMAT (3625), la Agencia Paulista de Tecnologia em Agronegocios, Polo Regional Nordeste Paulista (3557), North Carolina State University, Departamento de Ciencia do Solo (3523), CIRAD Prifas (con 3381), APTA Regional Centro Leste (3376), Embrapa Meio Norte y Universidade Federal do Piaui ambos con 3302 puntos, EMBRAPA Amazonia Ocidental, Municipio Aripuana (MT) y Bundesforsch Anstalt Forst \& Holzwirstchaft todos ellos con 3299 puntos, Laboratorio de Bioquímica Jardim Paulista, en su Departamento de Biología Molecular con 3297, y finalmente INCRA MT e INCRA Cuiabá, ambos con 3285 puntos.

Dentro de las representaciones no se alcanza ningún sentido si no se consideran las prácticas aplicadas al análisis, primero con Google Maps y luego con Google Earth, si se quieren obtener varias formas para un mismo contenido. Por lo que se demuestra que la visualización en Google Earth no es la más adecuada para reflejar la representación de la UFMT. De manera que, si se empleó Google Earth, fue para identificar como es la representación a escala mundial a la hora de reflejar las firmas de colaboración de una institución con el resto según los criterios de los geo-mapas.

Para Google Earth la forma de trabajar una red social se enfoca a partir de un archivo en .kml, donde las relaciones se muestran por coordenadas de longitud y latitud, de lo que resulta una visualización gráfica muy agradable, como se aprecia en la Figura 3.

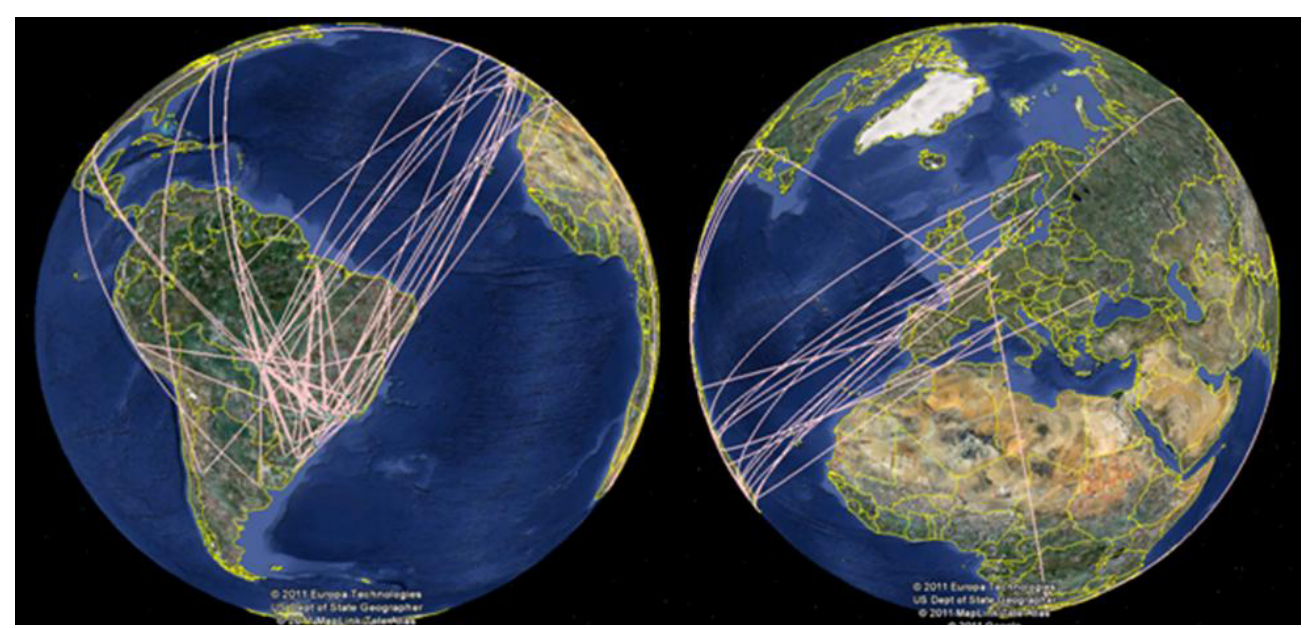

Figura 3. Distribución de las relaciones de la UFMT en el Google Earth con datos de la Web of Science.

Todas las relaciones de la UFMT quedan mejor explicadas si el análisis se hace mediante Google Maps. Pues ofrece una forma mejor de percibir la composición real del estatus científico en el medio investigador. Así pues, la distribución se caracteriza mejor si 
se emplea Google Earth, al ofrecer vertientes distintas a la hora de visualizar un mismo contenido, lo que tiene mejores posibilidades innovadoras.

\section{CONSIDERACIONES SOBRE LA REPRESENTACIÓN DE LA UFMT DENTRO DEL MUNDO INVESTIGADOR.}

De todo ello se infiere que, a la hora de mostrar estas colaboraciones, en especial si se las representa con los citados productos de Google, las instituciones de investigación cuentan con un recurso innovador para conocer su comportamiento de facto en relación a sus pares científicos, en este caso aplicado a representar la UFMT. En especial, resulta muy adecuado identificar como fueron esas relaciones empleando estos soportes informacionales.

Surge así la representación del comportamiento cualitativo de la UFMT, su interacción y articulación dentro de un escenario mundial, donde el tamaño de los nodos que indican la frecuencia en el análisis se separó por grupos, con lo que se identificaron siete grupos principales por áreas de trabajo con otras universidades.

Para las Ciencias de la Computación, muestra una gran consolidación en relación con la Universidade de São Paulo (latitud de la USP -23.56218: Longitud de la USP 46.72336).

Tratandose de la Física las principales instituciones fueron: la Universidade Estadual de Maringá (latitud -23.41030: longitud -51.93800), Unicamp (latitud -23.11805: longitud -49.50325), Universidade Federal de Goiás (latitud -9.27562: longitud -46.84570), Hokkaido University (latitud 68.65655: -132.89063), Universidade de São Paulo (latitud 23.56218: longitud -46.72336), UFSC (latitud -19.22818: longitud -49.74609), Aarhus University (latitud 56.12947: longitud 9.02705), Florida State University (latitud 48.45835: longitud -36.91406), Max Planck Institute for Solid State Resource (latitud 67.47492: longitud -8.08594), Darmstadt University of Technology (latitud 68.13885: longitud -1.05469) entre otras buenas instituciones de investigación.

Sobre el área de Química tuvimos la Universidade Estadual de Londrina con (latitud 23.32707: longitud -51.19907), seguida por la Universidade Federal de São Carlos (latitud -19.84939: longitud -48.77930), IPEN/CNEN/SP (latitud -23.56218: longitud -46.72336), Unicamp (longitud -23.11805: longitud -49.50325), CNR Istitute Geocronology \& Geochemical Isotopes Pisa (latitud 75.35190: longitud -20.39063), Universidade Federal do Ceará (latitud 56.94497: longitud -20.39063), Unesp (-4.91583: -43.41797), Universidade Federal da Bahia (latitud 51.17934: longitud -20.39063).

En el área de Agricultura y Zootecnia, las cooperaciones con más destaque fueron la Escola Superior de Agricultura "Luiz de Queiroz" con (latitud 76.51682: longitud 8.43750), Universidade Federal Rural do Rio de Janeiro (latitud 12.89749: longitud 39.02344), Universidade Estadual de Maringá (latitud -23.41030: longitud -51.93800), Unesp (latitud -4.91583: longitud -43.41797), Universidade Federal de Viçosa (latitud 20.30342: longitud -36.56250), Unemat (latitud 6.31530: longitud -44.12109), 
Universidade Federal de Alagoas (latitud 8.58102: longitud -33.39844), Universidade Federal de Pernambuco (latitud 1.05463: longitud -32.60742), Universidade de Brasília (latitud -14.67925: longitud -47.74658), Cornell University (latitud 54.47004: longitud 77.16797), Duke University (latitud 49.38237: longitud -83.84766), Universidade Federal do Rio de Janeiro (latitud -21.81051: longitud -43.07739), Unicamp (latitud -23.11805: longitud -49.50325), Universidade Federal de Lavras (latitud -15.62304: longitud 34.54102), University Conpenhagen (latitud 71.52491: longitud 47.46094) y California State University (latitud 58.07788: longitud -105.46875).

Para las Ingeniería y Ciencia de los Materiales, las principales relaciones fueron con la Universidade Federal do Rio Grande do Sul (latitud -25.99755: longitud-48.69141), Technical University of Denmark (latitud 62.91523: longitud 29.53125), Unesp (4.91583: -43.41797), Universidade de São Paulo (latitud -23.56218: longitud -46.72336) y IPEN/CNEN/SP (-23.56218: -46.72336).

Para las áreas del ámbito de la salud Medicina y Veterinaria, las relaciones más intensas fueron con la Universidade Federal de Viçosa (latitud 20.30342: longitud 36.56250), Universidade Federal de Lavras (latitud -15.62304: longitud -34.54102), Universidade de São Paulo (latitud -23.56218: longitud -46.72336), Unesp (latitud 4.91583: longitud -43.41797), Universidade de Brasília (latitud -14.67925: longitud 47.74658), Cornell University (latitud 54.47004: longitud -77.16797), Unicamp (latitud 23.11805: longitud -49.50325), University of Manchester (latitud 62.99516: longitud 2.63672), Medine College Winsconsin (latitud 54.97761: longitud -82.26563), Unifesp (latitud -19.35261: longitud -45.17578), Instituto FioCruz (latitud -19.39407: longitud 45.13184) y University of British Columbia (latitud 59.80063: longitud -113.55469).

Se aprecia con claridad en todas las relaciones que los asuntos (áreas de estudio) de interés más regional se suelen realizar en colaboración con instituciones nacionales (brasileñas), sin embargo las de carácter internacional y más inmediatas se hacen mediante firmas de colaboración internacional, como sucede en las áreas de agricultura y zootecnia. Un claro ejemplo de esto son las ciencias de la salud, donde la Universidade Federal de Mato Grosso (UFMT) ha alcanzado un estatus de referencia mundial en enfermedades tropicales; también en cuanto se refiere al cultivo de soja, de algodón y de maíz, asimismo en la zootecnia de ganado bovino y cebuino, pues este estado brasileño es uno de los mayores productores de carne vacuna.

El área de física se caracteriza por ofrecer una interacción intermediaria, mientras que las ciencias materiales, ingenierías, ciencia de la computación y química trabajan cosas más primarias en escalas de representación científica.

\section{CONCLUSIONES.}

De manera que parece confirmarse que el rendimiento de la colaboración científica en el Estado de Mato Grosso no ha sido elevada desde la perspectiva internacional. Lo que no impide que haya alcanzado un desempeño interesante en el interior de la federación 
brasileña, al mostrar una buena colaboración con la USP, la Universidade Federal de Viçosa y la Universidade Federal de Lavras especialmente.

En términos de visibilidad cabría esperar que su representación hubiese estado restringida a lo local, pero no fue lo que sucedió. Otro aspecto destacable ha sido el impulso recibido por la gran aportación financiera del gobierno brasileño desde 2002, con una inversión de recursos considerable para la mejora de los programas de postgrado en el estado y, sobre todo, en la UFMT.

Aparecen como los principales contribuyentes de la UFMT la Unemat y la Unic. Estas instituciones aparecen en los índices casi siempre como autores secundarios.

Las áreas clave dentro de la UFMT están compuestas por los siguientes clusters de dominios científicos:

- Medicina, con su foco principal en Medicina Tropical. Si bien aparecen reflejadas con intensidad en el Estado otras subáreas como la salud laboral, medicina de familia, radiología, medicina nuclear, medicina quirúrgica y veterinaria;

- Agricultura, con fuerte influencia de los estudios sobre cultivos y conservación de la flora en el cerrado, la ecología, la biodiversidad, la agronomía, la horticultura, la botánica y la geología;

- Ingenierías, como consecuencia de la implantación de nuevas titulaciones, pues han supuesto una apertura en la innovación dentro del Estado. De tal manera que ha llevado a la UFMT a dar una imagen más moderna y vanguardista. Este efecto ya se percibe en los resultados de la producción científica;

- Aunque la Física y Química son vitales para la consolidación de una universidad de prestigio, sin embargo en la UFMT no alcanzan una gran productividad, por lo que sus relaciones no tienen un reflejo muy representativo;

- Las áreas tecnológicas, como la informática, también presentan un índice bajo, igual que la Zoología, las áreas de humanidades y las ciencias sociales aplicadas.

El reflejo de todas las colaboraciones y dimensiones de su representación se hace de acuerdo con las relaciones que muestran sus estudios, sin embargo cuando la producción es baja sus colaboraciones se muestran también con escasa intensidad.

Para la aportación metodológica de la información en representación espacial hace con que sea posible mirar, en ámbito geográfico, como las instituciones hacen sus colaboraciones científicas con mejor visualización de la información, pues todo el propósito de la investigación fue la forma de su representación. 


\section{NOTAS.}

${ }^{1}$ Plataforma de mapas informacionales, con estructura para generar descripción para el Google Maps. Disponible en: $<$ http://www.arcgis.com/about/>. [Consulta: enero de 2013].

${ }^{2}$ Software para descripción de datos de latitud y longitud. Disponible en: $<$ http://www.gpsvisualizer.com/ $>$. [Consulta: enero de 2013].

${ }^{3}$ Cameo's es cuando una persona importante hace apariciones en un determinado programa. Para la ciencia los Cameo's se dan por trabajos aislados de grandes personajes en trabajos que no tratan específicamente de sus temas principales, siempre como autores secundarios.

\section{BIBLIOGRAFÍA.}

BEZERIANOS, A.; CHEVAlIER, F.; DRAGICEVIC, P.; ELMQVIST, N. y FEKETE, J.D. GraphDice: A System for exploring Multivariate Social Networks. Eurographics / IEEE-VGTC Symposium on Visualization, 2010, vol. 29, $\mathrm{n}^{\circ} 3, \mathrm{p} .863-872$.

BIGHAM, J.M. et al. Geocoding police collision report data from California: a comprehensive approach. International Journal of Health Geographics, 2009, vol. 8, $\mathrm{n}^{\mathrm{o}} 72$, p. 1-10.

CHIN WU, I. y YING WU, C. Using internal link and social network analysis to support searches in Wikipedia: A model and its evaluation. Journal of Information Science, 2011, vol. 37, n 2, p. 189-207.

CROSBY, A.W. The Measure of Reality: Quantification and Western Society. Cambridge, New York. 1997.

FARMAN, J. Mapping the digital empire: Google Earth and the process of postmodern cartography. New Media \& Society, 2010, vol. 12, nº 6, p. 869-888.

FREEEMAN, L.C. Visualizing Social Networks. Journal of Social Structure [en línea]. 2000. Disponible en: $<$ http://citeseerx.ist.psu.edu/viewdoc/download?doi=10.1.1.87.6279\&rep=rep1\&type=pd f $>$. [Consulta: 12 de junio de 2011].

HOLBROOK, J.A.D. Why measure science? Science and Public Policy, 1992, vol. 19, n 5, p. 262-266.

HOPPE, B. y REINELT, C. Social network analysis and the evaluation of leadership networks. The Leadership Quarterly, 2010, vol. 21, p. 600-619.

LEYDESDORFF, L. y PERSSON, O. Mapping the geography of science: distribution patterns and networks of relations among cities and institutes. Journal of the American Society for Information Science and Technology, 2010, vol. 61, n $\mathrm{n}^{\mathrm{O}}$ 8, p. 1622-1634.

SANZ-CASADO, E. y MARTÍN MORENO, C. Aplicación de técnicas bibliométricas a la gestión bibliotecaria. Investigación Bibliotecológica, 1998, vol. 12, no 24, p. 24-40.

TURUGET, D. Sociología de la ciencia: bibliometría, cientometría y cientografía. Noticias para Químicos, 1989, no 316, p. 4-12. 
VELHO, L.M. Leme Strini. Indicadores científicos. Interciencia, 1990, vol. 15, n 3, p. $139-145$

VELHO, L.M. Leme Strini. Indicadores científicos: aspectos teóricos y metodológicos e impactos en la política científica. In: MARTÍNEZ, E. y ALBORNOZ, M. (ed.). Indicadores de ciencia y tecnología: estado del arte e perspectivas. Caracas: Nova Sociedad, 1998, p. 25-51.

WHITAKER, F. Redes: uma estrutura alternativa de organização. Rio de Janeiro: Rits, 1998.

WHITE, H.D. Author-centered bibliometrics through CAMEOs: Characterizations automatically made and edited online. Scientometrics, 2001, vol. 53, nº 3, p. 607-637.

YANG, B.; LIU, Z. y MELOCHE, J.A. Visualization of the Chinese academic web based on social network analysis. Journal of Information Science, 2010, vol. 36.

Anales de Documentación, 2013, vol. 16, nº 1 